\title{
Weight-Bearing and Effort Distributions at the Lower Limbs during the Five-Repetition Sit-to-Stand Test in Hemiparetic and Healthy Individuals
}

\author{
Anabèle Brière, ${ }^{1,2}$ Sylvie Nadeau,, ${ }^{1,2}$ Séléna Lauzière,, ${ }^{1,2}$ and Denis Gravel ${ }^{1,2}$ \\ ${ }^{1}$ Laboratoire de Pathokinésiologie, Centre de Recherche Interdisciplinaire en Réadaptation, Institut de Réadaptation \\ Gingras-Lindsay-de-Montréal, 6300 Avenue Darlington, Montréal, QC, Canada H3S 2J4 \\ ${ }^{2}$ École de Réadaptation, Faculté de Médecine, Université de Montréal, C.P.6128 Succ. Centre-Ville, Montréal, QC, Canada H3C 3J7
}

Correspondence should be addressed to Anabèle Brière, anabele.briere@umontreal.ca

Received 5 July 2011; Accepted 14 September 2011

Academic Editor: C. I. Renner

Copyright (C) 2012 Anabèle Brière et al. This is an open access article distributed under the Creative Commons Attribution License, which permits unrestricted use, distribution, and reproduction in any medium, provided the original work is properly cited.

\begin{abstract}
Background. The weight-bearing (WB) and effort distributions during the five-repetition sit-to-stand test (5R-STS) were assessed in healthy and hemiparetic subjects and were compared to the distributions obtained for a single STS task (1-STS). Methods. Eighteen hemiparetic subjects and 12 controls were included. The WB distribution and time were computed using the vertical ground reaction forces. The knee muscles' effort distribution was quantified with the electromyographic (EMG) data of the STS transfers expressed relatively to the EMG values of maximal strength assessments. Results. In both groups, the time, WB, and effort distributions did not differ between repetitions of the 5R-STS test. The WB and effort distributions of the first repetition were more asymmetrical than those for the 1-STS for the hemiparetic subjects only. Conclusions. Since no changes were found between repetitions, the 5R-STS test might not be demanding enough. The hemiparetic subjects adopt different WB and effort distribution strategies according to the number of STSs to complete.
\end{abstract}

\section{Introduction}

Initially introduced as a measure of lower limb strength $[1,2]$, the five-repetition sit-to-stand (5R-STS) test is also used to assess balance [3], fall risk [4], and the effect of interventions to improve balance, muscle strength, or mobility in various populations $[5,6]$. In subjects with chronic stroke, the 5R-STS test has also been used to evaluate functional performance [7].

Following a stroke, STS and stand-to-sit transfers are performed with an asymmetrical weight-bearing (WB) distribution with more weight usually taken by the nonparetic limb $[8,9]$. The knee extensor joint moments are also asymmetrical with greater moments produced by the nonparetic side [10]. Interestingly, except for measuring the time to complete the 5R-STS test, no study to date has tested the effect of repeating consecutive STS transfers on WB and on the muscular demand at the knee in healthy and physically impaired participants such as poststroke survivors. This would help to clarify how WB and effort distributions are managed in more demanding situations involving repeated lower limb muscular efforts. The idea being that knowing the number of repetitions to be completed might change the motor strategy in order to consider the additional effort needed to complete the task. The objectives of this study were to (1) assess the association between time to execute the 5R-STS test and WB distribution and the effort distribution at the knees; (2) determine whether time, WB distribution, and effort distribution at the knees differ among the 5R-STS repetitions; (3) examine if $\mathrm{WB}$ and effort distribution strategies vary with the number of repetitions to be performed (1 versus 5). For hemiparetic individuals, it was hypothesized that time to execute the 5R-STS would be associated with WB and effort distributions: the slowest subjects being the most asymmetrical. They will present asymmetrical WB and effort distributions for the first cycle of the 5R-STS test, and these asymmetries as well as the time cycle will increase from the first to the fifth. A task such as executing five consecutive STSs 
should be demanding enough for this population to increase these asymmetries. Finally, the WB and effort distributions of hemiparetic individuals will differ between a single STS (1-STS) and the first cycle of the 5R-STS test. For healthy individuals, these factors will not affect their performance.

\section{Materials and Methods}

2.1. Participants. Eighteen chronic hemiparetic individuals (3 to 27 years poststroke; 10 with a left hemiparesis) able to rise from a standard-height chair $(45$ to $50 \mathrm{~cm}$ ) without using their arms and 12 elderly controls participated in this cross-sectional study (Table 1). The hemiparetic individuals had a moderate level of motor impairment (mean score of 5.2/7 $( \pm 1.1)$ for the leg and 3.7/7 $( \pm 1.3)$ for the foot on the Chedoke McMaster Stroke Assessment [11]). One rated his pain level in the lower limbs at rest as 5.5/10 on the $10 \mathrm{~cm}$ visual analog scale (VAS), [12] and six rated their pain level on activity between 2.6 and 5.7/10; the others presented a pain level of 2 or less. Except for two that had an hypnoanesthesia at the foot (unable to detect the 6.65 Semmens-Weinstein monofilament [13]), and one showing a severe sensory deficit (unable to feel the 5.18 monofilament), the others had good tactile sensation. All had a good sense of vibration and position evaluated with a $128 \mathrm{~Hz}$ tuning fork and "up or down" segment position of the foot, respectively [13]. They presented moderate spasticity at the ankle (mean score of 8.7/16 on the Index of Spasticity of Levin and Hui-Chan [14]). Their balance was good (Berg Balance Scale [15] score > 47), except for one that had a score of 40 . Their mean natural and maximal walking speeds over $5 \mathrm{~m} \mathrm{[16]} \mathrm{were} 0.78 \mathrm{~m} / \mathrm{s}( \pm 0.25)$ and $1.10 \mathrm{~m} / \mathrm{s}$ $( \pm 0.35)$, respectively. The nonparetic side was stronger than the paretic side $(170.3 \pm 58.8 \mathrm{Nm}$ versus $119.4 \pm 52.9 \mathrm{Nm} ; P<$ 0.001; Table 1). Participants were excluded if they presented with comprehensive aphasia, cognitive deficit (Folstein MiniMental exam [17] score < 25/30) and non-stroke-related disabilities. The subjects in the control group had no health problems and were recruited among the employees of a rehabilitation centre (Institut de réadaptation Gingras-Lindsayde-Montréal) where the laboratory assessments were also performed. Subjects gave written informed consent. This experiment was approved by the local ethics committee and followed institutional guidelines.

2.2. Maximal EMG at the Knee. A dynamometric assessment with a Biodex dynamometer (Biodex Medical Systems, New York, USA) followed the clinical assessment and allowed the maximal EMG data (denominator) needed to quantify the levels of effort at the knee to be determined. This was obtained by quantifying the Electromyographic Muscular Utilization Ratio (EMUR, [18]), that is, the ratio relating the EMG of the knee muscles collected during the STS transfers (numerator) to the maximal EMG obtained during maximal strength assessments (denominator). The knee extensors were chosen because they present high levels of effort during the STS transfer $[10,19]$.

Following appropriate skin preparation [20], EMG bipolar surface electrodes ( $20 \mathrm{~mm}$ interelectrode distance) were placed bilaterally on the skin over the vastus lateralis, vastus medialis, and rectus femoris. Electrogoniometers, placed on the lateral face of both knees, provided a common reference for the knee positions between the STS and the dynamometric assessments. Muscle activity was acquired with a 16channel EMG telemetric system (Noraxon Telemyo System). Standard methods of surface recording [20] were used to record amplified signals at $1200 \mathrm{~Hz}$ with a customized Labview program. The subjects were stabilized with straps in a sitting position with the hips flexed $60^{\circ}$. Since the greatest levels of effort during the STS are generally found at seat-off when the knees are in a $75^{\circ}$ flexion position $[10,19]$, this angle was chosen to test the knee extensors' static maximal strength and record their maximal EMG. The mean of two trials $(<10 \%$ difference) was used to determine the maximal strength and EMG of the knee extensors.

2.3. Sit-to-Stand Task Assessment. Following the dynamometric assessment, a height-adjustable chair instrumented with force plates under each thigh was used to assess the STS tasks (details in Brière et al. [8]). Two AMTI (OR6-7-1000) force plates were used to record the forces under each foot. The EMG recorded during the tasks provided the numerator of the EMUR and quantified the relative involvement of the knee muscles during the STS tasks. The EMG and force plate signals were collected at $1200 \mathrm{~Hz}$ and $600 \mathrm{~Hz}$, respectively.

With arms crossed on their chest and feet placed in a spontaneous position, the subjects were asked to complete five consecutive STSs (5R-STS test) as fast as possible and instructed to stand up straight between repetitions. Timing started when the signal "Go" was given and ended when the subject's buttocks touched the chair on the fifth repetition. Subjects were also asked to complete a single STS at natural speed as they usually do (1-STS task). No instruction was given regarding the foot position (spontaneous). Two trials were executed.

2.4. Data Analysis. The vertical ground reaction forces (VGRF) were analyzed for all STS transfers. The WB distribution on each side, expressed in percent of total WB, was determined using the VGRF of the seat and foot averaged over a $1 \mathrm{~s}$ time interval (from $-0.5 \mathrm{~s}$ before seat-off to $0.5 \mathrm{~s}$ after; details in Brière et al. [8]). The seat-off corresponded to the time when the forces on the force plates of the seat were null. This interval was chosen because it covers the greatest efforts during the STS task [10]. The mean of two trials was used.

To determine the duration (seconds) of each cycle, the time elapsed between seat-off and seat-on (when the subject establishes contact with the seat) was computed. These events were extracted using the VGRF data of the seat.

To estimate the EMUR of the knee muscles, the EMG data corresponding to the maximal strength values of the knee extensors and to the seat-off had to be analyzed. To consider the electromechanical delay (generally ranging from 30 to $100 \mathrm{~ms}$ [21]) between the maximal electrical muscle activity value and the maximal strength value as well as the seat-off event, a time-window EMG analysis of $250 \mathrm{~ms}$ prior to the maximal strength value and to the seat-off event was determined. All raw EMGs were visually inspected 
TABLE 1: Characteristics of the subjects.

\begin{tabular}{lccc}
\hline Characteristics & Hemiparetics & Controls & $P<0.05$ \\
Mean \pm SD (range) & mean \pm SD (range) & 0.06 \\
Age, years & $58.8 \pm 11.9(35-83)$ & $1.7 \pm 0.1(1.7-1.9)$ \\
Height, $\mathrm{m}$ & $1.7 \pm 0.1(1.5-1.9)$ & $79.8 \pm 16.7$ & $(52.0-104.0)$ \\
Weight, $\mathrm{kg}$ & $80.2 \pm 24.0$ & $27.8 \pm 4.5(21.4-35.2)$ \\
Body mass index, kg/m & $(43.0-160.0)$ & $7 / 5$ & 0.96 \\
Male/female & $27.3 \pm 7.8(18.1-53.8)$ & $151.9 \pm 33.8$ \\
Knee extensor muscle strength, Nm & $14 / 4$ & $(88.8-206.9)$ \\
(nonparetic side or right side) & $170.3 \pm 58.8$ & $164.7 \pm 46.4$ \\
Knee extensor muscle strength, Nm & $(92.5-286.1)$ & $(92.7-234.1)$ \\
(paretic side or left side) & $119.4 \pm 52.9$ & 0.86 \\
\hline
\end{tabular}

then filtered with a 4 th-order Butterworth high-pass filter at $20 \mathrm{~Hz}$. Subsequently, a root mean square (RMS) was computed to quantify the amplitude of the EMG signal. The EMG ${ }^{\mathrm{rms}}$ value of each muscle collected during the task was then normalized $\left(\mathrm{EMG}_{\text {norm }}\right)$ to the maximal $\mathrm{EMG}^{\mathrm{rms}}$ value obtained during the strength testing at the same knee angle $\left(75^{\circ}\right)$ to estimate the EMUR. Subsequently, the mean of the EMUR of the vastus medialis, vastus lateralis, and rectus femoris was computed so as to have an EMUR for the extensors, which better represents the global effort at the knees. The mean of two trials was used.

Furthermore, to interpret the effort in terms of asymmetry between sides, the extensors' EMUR on each side had to be expressed in percent of total bilateral effort as done for the WB distribution. Therefore, an Index of asymmetry of EMUR was computed as follows:

$$
\frac{\text { EMUR (nonparetic side) }}{\text { EMUR (nonparetic side) + EMUR (paretic side) }} * 100 .
$$

Some EMG data were eliminated due to the presence of noise in the recorded signal. This represented 8/98 signals for the 1-STS task. For the 5R-STS test, the EMG data of the rectus femoris and of the vastus lateralis had to be removed in 11 subjects ( 5 hemiparetics, 6 controls) and 5 subjects ( 2 hemiparetics, 3 controls), respectively. For the statistical analyses, the indices of asymmetry of effort and the WB distribution values of the nonparetic (hemiparetics) and right sides (controls) were used.

2.5. Statistical Analyses. Descriptive statistics were computed on all variables. The conditions for using parametric tests were fulfilled. In both groups, Pearson correlation coefficients were used to assess the associations between time to execute the 5R-STS test and WB and effort distributions. Also, as previous studies [8, 9] have already established that hemiparetic individuals perform more asymmetrically than healthy ones, and as the two groups here did not have the same number of subjects, the group effect was not considered in the further analyses. Therefore, three one-way repeated-measures ANOVAs tested the effect of consecutive STSs (repeated factor) on WB distribution, effort distribution, and time cycle, respectively, separately in each group. If these ANOVAs showed any significant values $(P<$ 0.05), pairwise comparisons were made to establish the sites of differences using an appropriate Bonferroni correction $(0.05 / 10$ contrasts $=0.005)$. To determine if WB and effort distributions strategies varied according to the number of repetitions paired Student's $t$-tests were completed for both groups separately between the WB and effort distributions obtained for the 1-STS task and those found for the first repetition of the 5R-STS test. All statistics were performed using the 13th version of SPSS package software, and the level of significance was fixed at 0.05 , except for those corrected for the number of comparisons.

\section{Results}

3.1. Association between Time to Execute the 5R-STS Test, WB Distribution, and Effort Distribution at the Knees. The duration of the 5R-STS was longer for the hemiparetic individuals than for the healthy participants $(19.3 \mathrm{~s}( \pm 5.3)$ versus $11.1 \mathrm{~s}( \pm 1.7) ; P<0.05)$. No association was found between the time to execute the 5R-STS test and the WB or the effort distribution at the knees.

3.2. Effect of 5 Consecutive Repetitions on Time Cycle, WB Distribution, and Effort Distribution at the Knees. For the time cycle, WB distribution and effort distribution at the knees, the ANOVAs did not show any significant difference between the 5 repetitions for the hemiparetic or healthy individuals (Table 2) (ANOVAs; $P>0.05$ ).

3.3. Comparison of WB and Effort Distribution Strategies with Number of Repetitions to Be Performed (1 versus 5). The WB $(59.2 \% \pm 6.1 \%)$ and effort $(58.7 \% \pm 10.8 \%)$ distributions of the first repetition of the 5R-STS test were more asymmetrical $(P=0.001$ and $P=0.04$, resp. $)$ than those obtained for the 1-STS task for hemiparetic subjects (WB: $56.1 \% \pm 5.5 \%$; effort: $56.3 \% \pm 11.4 \%$ ) (Table 2; Figure $1(\mathrm{a})$ ). For the controls, no difference was found between the WB $(49.1 \% \pm 4.1 \%)$ and effort $(51.0 \% \pm 7.2 \%)$ distributions of the first repetition of the 5R-STS test and those measured for the $1-S T S$ (WB: $49.7 \% \pm 2.9 \%$; effort: $51.1 \% \pm 8.3 \%$ ) 
TABLE 2: Mean and standard deviation values of the time, WB, and effort distributions for the 1-STS task and the five cycles (C1-C5) of the 5R-STS test for the hemiparetics and the controls (nonparetic and right sides, resp.).

\begin{tabular}{|c|c|c|c|c|c|c|c|}
\hline \multirow{2}{*}{ Groups } & \multirow{2}{*}{ Variables } & \multirow{2}{*}{ 1-STS } & \multicolumn{5}{|c|}{ 5R-STS } \\
\hline & & & $\mathrm{C} 1$ & $\mathrm{C} 2$ & C3 & $\mathrm{C} 4$ & C5 \\
\hline \multirow{3}{*}{ Hemiparetics } & Time (s) & - & $2.5(0.8)$ & $2.4(0.6)$ & $2.3(0.6)$ & $2.3(0.7)$ & $2.4(0.7)$ \\
\hline & WB distribution (\%) & $56.1(5.5)$ & $59.2(6.1)$ & $59.1(6.7)$ & $58.9(7.0)$ & $59.2(7.9)$ & $60.1(7.7)$ \\
\hline & Effort distribution (\%) & $56.3(11.4)$ & $58.7(10.8)$ & $58.7(10.5)$ & $59.3(9.3)$ & $59.1(10.5)$ & $58.7(9.8)$ \\
\hline \multirow{3}{*}{ Controls } & Time $(\mathrm{s})$ & - & $1.4(0.3)$ & $1.4(0.2)$ & $1.3(0.2)$ & $1.3(0.2)$ & $1.3(0.3)$ \\
\hline & WB distribution (\%) & $49.7(2.9)$ & $49.1(4.1)$ & $49.8(2.9)$ & $50.3(3.6)$ & $50.9(3.8)$ & $50.8(3.8)$ \\
\hline & Effort distribution (\%) & $51.1(8.3)$ & $51.0(7.2)$ & $51.9(8.5)$ & $49.6(8.0)$ & $51.8(8.0)$ & $54.2(8.3)$ \\
\hline
\end{tabular}

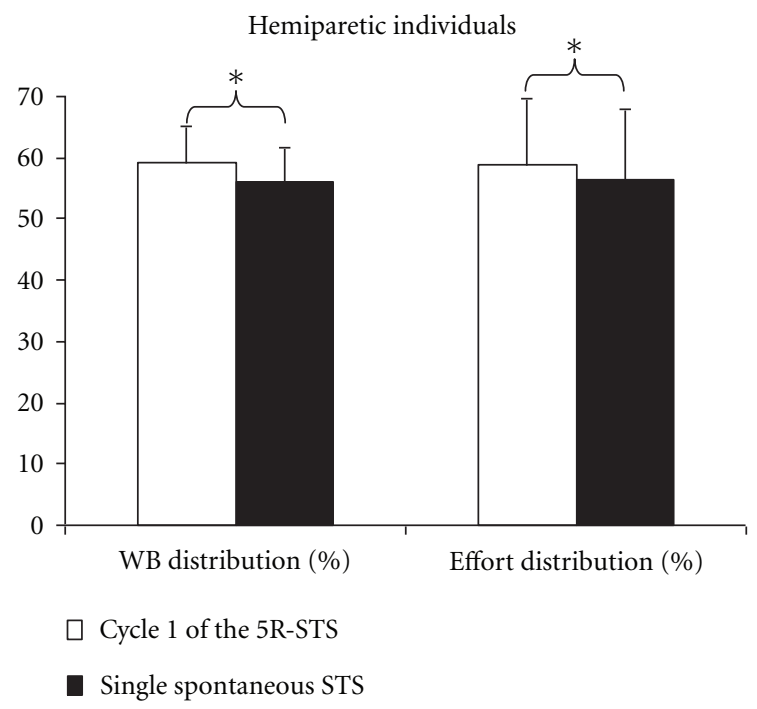

(a)

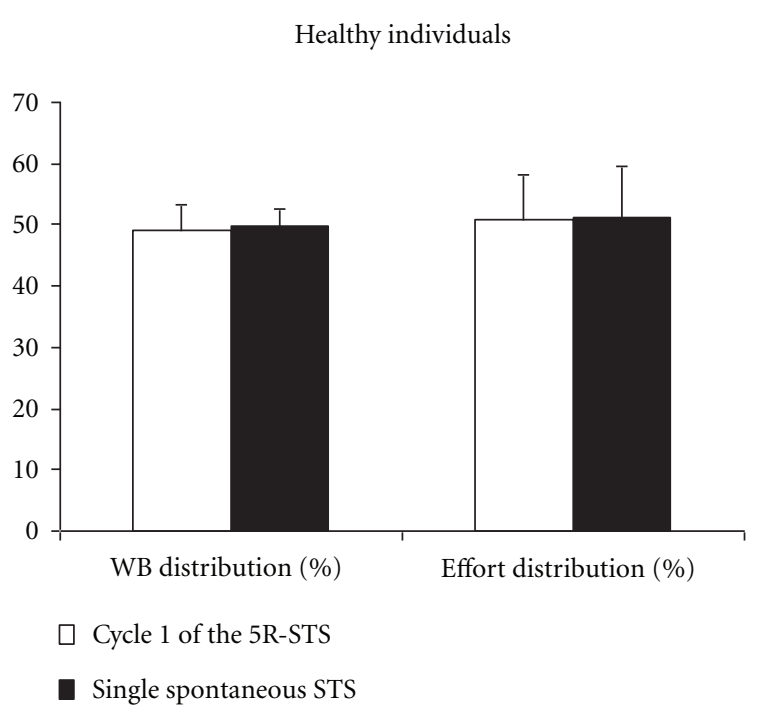

(b)

FIGURE 1: Graphs showing the mean distributions (\%) of weight bearing (WB) and effort at the knees for the first cycle of the 5-repetition sit-to-stand test (5R-STS) (in white) and the single sit to stand executed at natural speed in a spontaneous foot position (in black) for hemiparetic individuals (a) and healthy individuals (b). Vertical lines represent the one-standard deviation. Asterisks indicate significant differences between the two conditions $(P<0.05)$.

(Table 2; Figure 1(b)). Scatter plots of associations between these two tasks for the WB and the effort distributions show clearly the shift under the line of identity for the hemiparetic group illustrating their more asymmetrical strategy for completing the 5R-STS test (Figures 2(a) and 2(b)). For the controls, these scatter plots show that WB and effort distributions spread fairly evenly over and under the line of identity (Figures 2(c) and 2(d)).

\section{Discussion}

The first important finding is that the subjects most asymmetrical in their WB or effort distributions are not the slowest in the 5R-STS. Having a very asymmetrical WB or effort distribution when doing a STS transfer therefore does not imply that more time will be needed to complete the 5RSTS test and vice versa. In everyday life, standing up quickly is probably more important than performing a symmetrical sit to stand. Also, this result agrees with conclusions reported by Lord et al. [22] and by McCarthy et al. [23] concerning the multidimensional aspect of the STS transfer. According to these studies $[22,23]$, lower limb strength is not the only factor influencing STS performance but many other variables (e.g., balance, psychological, and sensorimotor parameters) or other strength-related parameters (e.g., rate of force development, and power) [24] could also influence the STS test completion time.

The second finding revealed that the participants are able to perform five consecutive STS without modifying their strategies. Therefore, for stroke participants, performing five consecutive STS is not challenging enough to produce a change in their motor performance. It would be interesting to assess what happens with the 30-second chair STS test [23] involving more repetitions.

A third interesting finding is that stroke subjects seemingly adopt different WB and effort distribution strategies according to the number of STSs to complete. Knowing the number of repetitions to be performed might trigger 


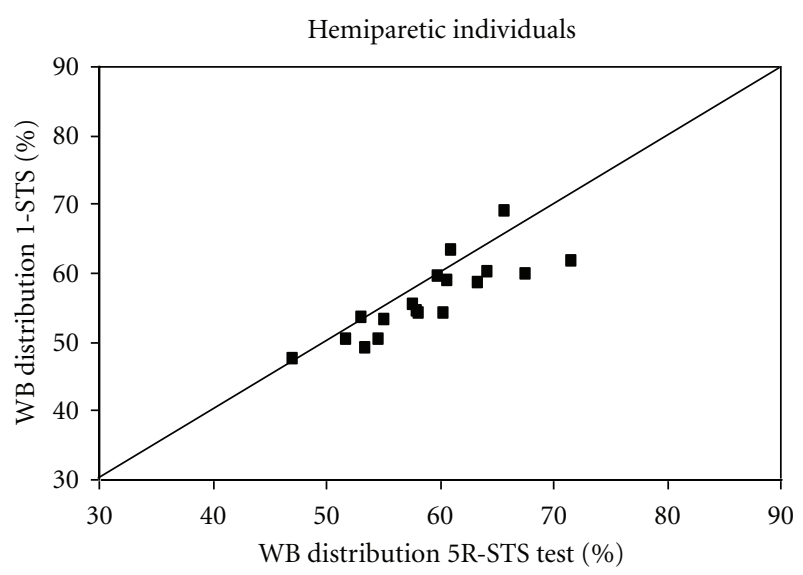

(a)

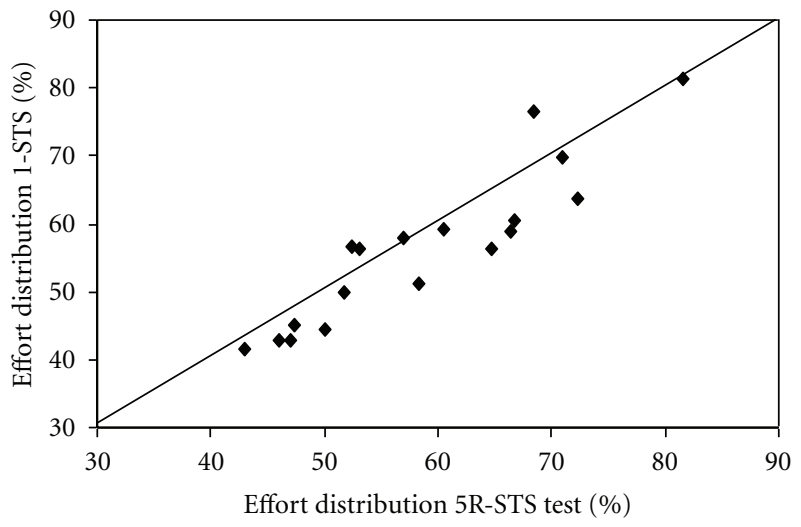

(b)

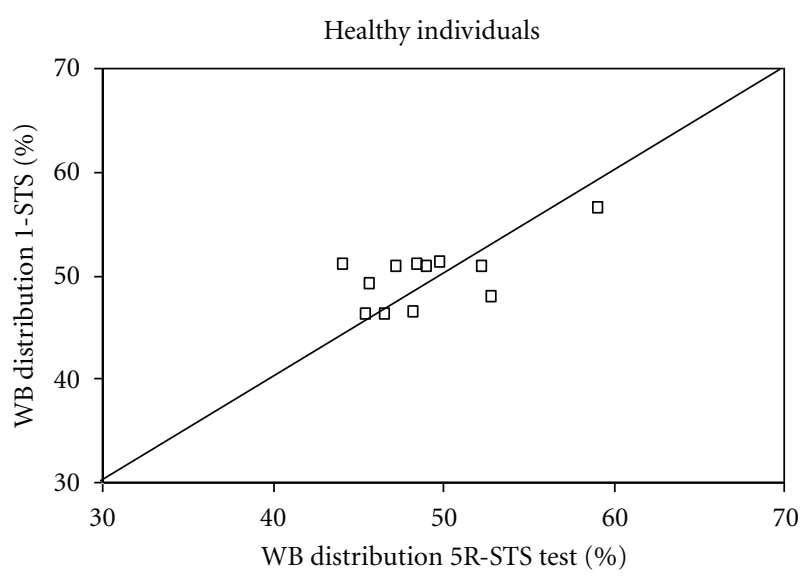

(c)

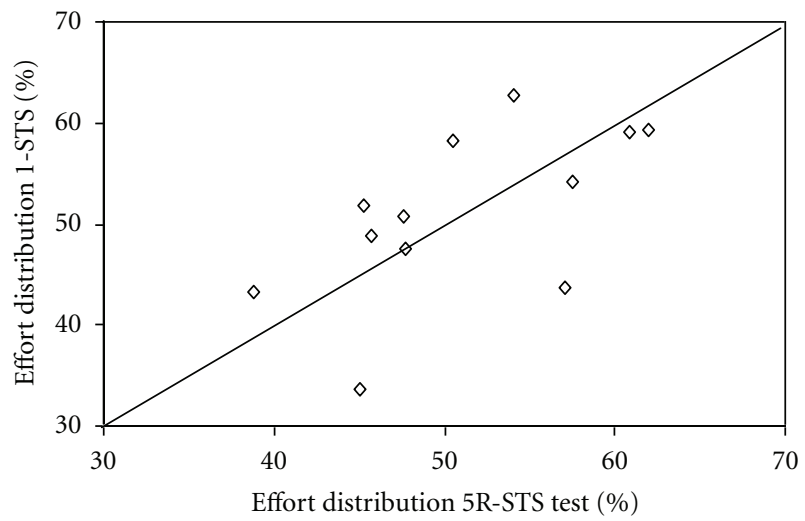

(d)

Figure 2: Scatter plots showing the associations between the weight-bearing (WB) distribution (squares) and the effort distribution (diamonds) for the first cycle of the 5-repetition sit-to-stand test (5R-STS; X axis) and those for the single sit-to-stand task (1-STS) for the hemiparetic individuals ((a) and (b), resp.) and the healthy individuals ((c) and (d), resp.). The lines of identity are presented.

reorganization of the lower limb effort and, thus, of the WB distribution. In fact, the anticipation of having more than one STS to do could explain the greater WB and effort on the nonparetic limb in the clinical test. A parallel could be made between this finding and the results of previous studies $[25,26]$ on associations between the distance covered by poststroke individuals during the 6- or 12-minute walk tests and their self-paced gait speeds established over 8 or 10 meters. In fact, they found that using these gait speeds to predict the distances walked in either 6 or 12 minutes overestimates the actual distances covered. Since the gait speeds were found to be relatively constant in the functional tests, the subjects seem to pace themselves more slowly even though the instructions given are to try to cover the greatest distance in 6 or 12 minutes $[25,26]$. Reorganization of the task execution might thus explain why the WB and effort distributions at the knees did not change with the repetitions. It would be interesting to see if the WB and effort distribution strategies remained the same without any instruction related to the number of repetitions preceding the 5R-STS test.

Finally, since the 5R-STS test has to be done as quickly as possible, the execution speed could also influence the strategy chosen by hemiparetic individuals. In comparison, the 1-STS task was executed at natural speed. Given that we did not do a stand-to-sit recording after the STS for the 1STS task, we cannot appreciate the influence of this factor. However, using data from the study by Duclos et al. [27], including comparable hemiparetic subjects and providing a mean time from seat-off to the end of the STS of $1.6 \pm 0.6 \mathrm{~s}$, it could be presumed that the mean time from standing to seat-on would be similar and, therefore, the mean time for spontaneous seat-off to seat-on would be close to that obtained in this study for the first cycle of the 5R-STS (3.2 versus $2.5 \mathrm{~s}$ ). Therefore, time is probably not a major issue when trying to explain the different behaviors between the 1-STS task and the 5R-STS test.

\section{Summary}

The results of this study revealed that the 5R-STS test is not demanding enough to induce time, $\mathrm{WB}$, and effort distribution variations between the repetitions. However, for the hemiparetic individuals, knowing the number of repetitions to be performed might have triggered an effort reorganization allowing them to perform 5 repetitions without modfying their WB and effort distribution strategies. 


\section{Disclosure}

An abstract including part of these results was submitted to the Canadian Stroke Congress 2011.

\section{Acknowledgments}

The OPPQ/REPAR partnership and the IRM Foundation funded this project. A, Brière and S. Lauzière were supported by a Ph.D. and a M.S. scholarship from the Fonds de la recherche en santé du Québec (FRSQ), respectively. Dr. S. Nadeau is a senior scientist supported by the FRSQ. The authors Michel thank Goyette and Daniel Marineau for their technical support; Susan Crabb and Julie Lecours for their collaboration in the data collection and the Cummings Jewish Centre for Seniors for their help with recruitment.

\section{References}

[1] M. Csuka and D. J. McCarty, "Simple method for measurement of lower extremity muscle strength," American Journal of Medicine, vol. 78, no. 1, pp. 77-81, 1985.

[2] R. W. Bohannon, "Alternatives for measuring knee extension strength of the elderly at home," Clinical Rehabilitation, vol. 12, no. 5, pp. 434-440, 1998.

[3] S. L. Whitney, D. M. Wrisley, G. F. Marchetti, M. A. Gee, M. S. Redfern, and J. M. Furman, "Clinical measurement of sit-tostand performance in people with balance disorders: validity of data for the Five-Times-Sit-to-Stand test," Physical Therapy, vol. 85, no. 10, pp. 1034-1045, 2005.

[4] B. Belgen, M. Beninato, P. E. Sullivan, and K. Narielwalla, "The association of balance capacity and falls self-efficacy with history of falling in community-dwelling people with chronic stroke," Archives of Physical Medicine and Rehabilitation, vol. 87, no. 4, pp. 554-561, 2006.

[5] J. M. Chandler, P. W. Duncan, G. Kochersberger, and S. A. Studenski, "Is lower extremity strength gain associated with improvement in physical performance and disability in frail, community-dwelling elders?" Archives of Physical Medicine and Rehabilitation, vol. 79, no. 1, pp. 24-30, 1998.

[6] C. Monger, J. H. Carr, and V. Fowler, "Evaluation of a homebased: exercise and training programme to improve sit-tostand in patients with chronic stroke," Clinical Rehabilitation, vol. 16, no. 4, pp. 361-367, 2002.

[7] A. Weiss, T. Suzuki, J. Bean, and R. A. Fielding, "High intensity strength training improves strength and functional performance after stroke," American Journal of Physical Medicine and Rehabilitation, vol. 79, no. 4, pp. 369-376, 2000.

[8] A. Brière, S. Lauziere, D. Gravel, and S. Nadeau, "Perception of weight-bearing distribution during sit-to-stand tasks in hemiparetic and healthy individuals," Stroke, vol. 41, no. 8, pp. 1704-1708, 2010.

[9] G. Roy, S. Nadeau, D. Gravel, F. Malouin, B. J. McFadyen, and F. Piotte, "The effect of foot position and chair height on the asymmetry of vertical forces during sit-to-stand and stand-to-sit tasks in individuals with hemiparesis," Clinical Biomechanics, vol. 21, no. 6, pp. 585-593, 2006.

[10] G. Roy, S. Nadeau, D. Gravel, F. Piotte, F. Malouin, and B. J. McFadyen, "Side difference in the hip and knee joint moments during sit-to-stand and stand-to-sit tasks in individuals with hemiparesis," Clinical Biomechanics, vol. 22, no. 7, pp. 795804, 2007.
[11] C. Gowland, P. Stratford, M. Ward et al., "Measuring physical impairment and disability with the Chedoke-McMaster stroke assessment," Stroke, vol. 24, no. 1, pp. 58-63, 1993.

[12] E. C. Huskisson, "Visual analogue scale," in Pain Measurements and Assessment, R. Melzack, Ed., pp. 33-37, Raven, New York, NY, USA, 1983.

[13] A. Thibault, R. Forget, and J. Lambert, "Evaluation of cutaneous and proprioceptive sensation in children: a reliability study," Developmental Medicine and Child Neurology, vol. 36, no. 9, pp. 796-812, 1994.

[14] M. F. Levin and C. W. Y. Hui-Chan, "Relief of hemiparetic spasticity by TENS is associated with improvement in reflex and voluntary motor functions," Electroencephalography and Clinical Neurophysiology, vol. 85, no. 2, pp. 131-142, 1992.

[15] K. Berg, S. Wood-Dauphinee, and J. I. Williams, "The balance scale: reliability assessment with elderly residents and patients with an acute stroke," Scandinavian Journal of Rehabilitation Medicine, vol. 27, no. 1, pp. 27-36, 1995.

[16] N. M. Salbach, N. E. Mayo, J. Higgins, S. Ahmed, L. E. Finch, and C. L. Richards, "Responsiveness and predictability of gait speed and other disability measures in acute stroke," Archives of Physical Medicine and Rehabilitation, vol. 82, no. 9, pp. 1204-1212, 2001.

[17] M. F. Folstein, S. E. Folstein, and P. R. McHugh, "'Mini mental state. A practical method for grading the cognitive state of patients for the clinician," Journal of Psychiatric Research, vol. 12, no. 3, pp. 189-198, 1975.

[18] L. J. Hébert, D. Gravel, and B. Arsenault, "Comparisons of mechanical and electromyographical muscular utilisation ratios," Scandinavian Journal of Rehabilitation Medicine, vol. 27, no. 2, pp. 83-88, 1995.

[19] T. Kotake, N. Dohi, T. Kajiwara, N. Sumi, Y. Koyama, and T. Miura, "An analysis of sit-to-stand movements," Archives of Physical Medicine and Rehabilitation, vol. 74, no. 10, pp. 10951099, 1993.

[20] R. Merletti and H. J. Hermens, "Detection and conditionning of the surface EMG signal," in Electromyography. Physiology, Engineering, and Noninvasive Applications, R. Merletti and P. Parker, Eds., pp. 107-131, John Wiley \& Sons, Hoboken, NJ, USA, 2004.

[21] P. R. Cavanagh and P. V. Komi, "Electromechanical delay in human skeletal muscle under concentric and eccentric contractions," European Journal of Applied Physiology and Occupational Physiology, vol. 42, no. 3, pp. 159-163, 1979.

[22] S. R. Lord, S. M. Murray, K. Chapman, B. Munro, and A. Tiedemann, "Sit-to-stand performance depends on sensation, speed, balance, and psychological status in addition to strength in older people," Journals of Gerontology A, vol. 57, no. 8, pp. M539-M543, 2002.

[23] E. K. McCarthy, M. A. Horvat, P. A. Holtsberg, and J. M. Wisenbaker, "Repeated chair stands as a measure of lower limb strength in sexagenarian women," Journals of Gerontology A, vol. 59, no. 11, pp. 1207-1212, 2004.

[24] C. G. Canning, L. Ada, and N. O’Dwyer, "Slowness to develop force contributes to weakness after stroke," Archives of Physical Medicine and Rehabilitation, vol. 80, no. 1, pp. 66-70, 1999.

[25] J. J. Eng, K. S. Chu, A. S. Dawson, C. M. Kim, and K. E. Hepburn, "Functional walk tests in individuals with stroke: relation to perceived exertion and myocardial exertion," Stroke, vol. 33, no. 3, pp. 756-761, 2002.

[26] C. M. Dean, C. L. Richards, and F. Malouin, "Walking speed over 10 metres overestimates locomotor capacity after stroke," Clinical Rehabilitation, vol. 15, no. 4, pp. 415-421, 2001. 
[27] C. Duclos, S. Nadeau, and J. Lecours, "Lateral trunk displacement and stability during sit-to-stand transfer in relation to foot placement in patients with hemiparesis," Neurorehabilitation and Neural Repair, vol. 22, no. 6, pp. 715-722, 2008. 


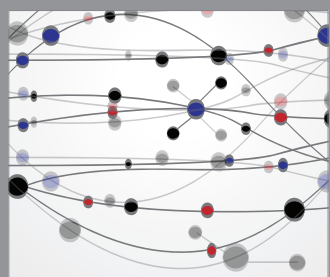

The Scientific World Journal
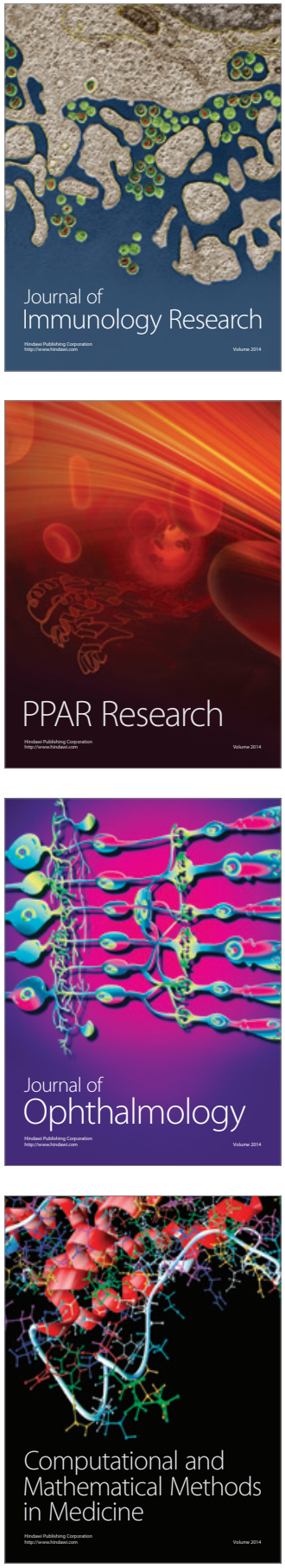

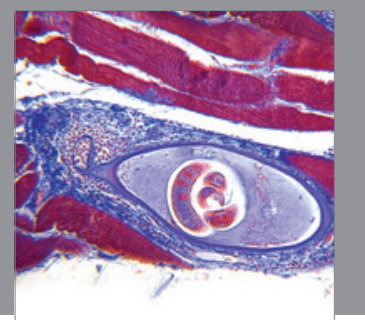

Gastroenterology

Research and Practice
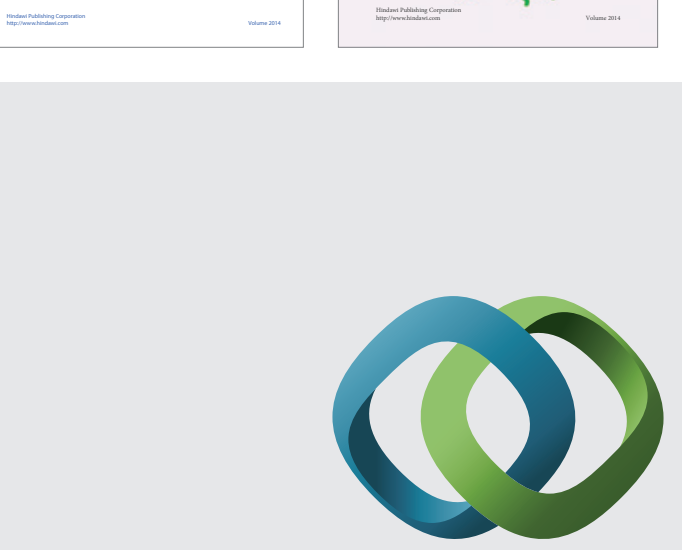

\section{Hindawi}

Submit your manuscripts at

http://www.hindawi.com
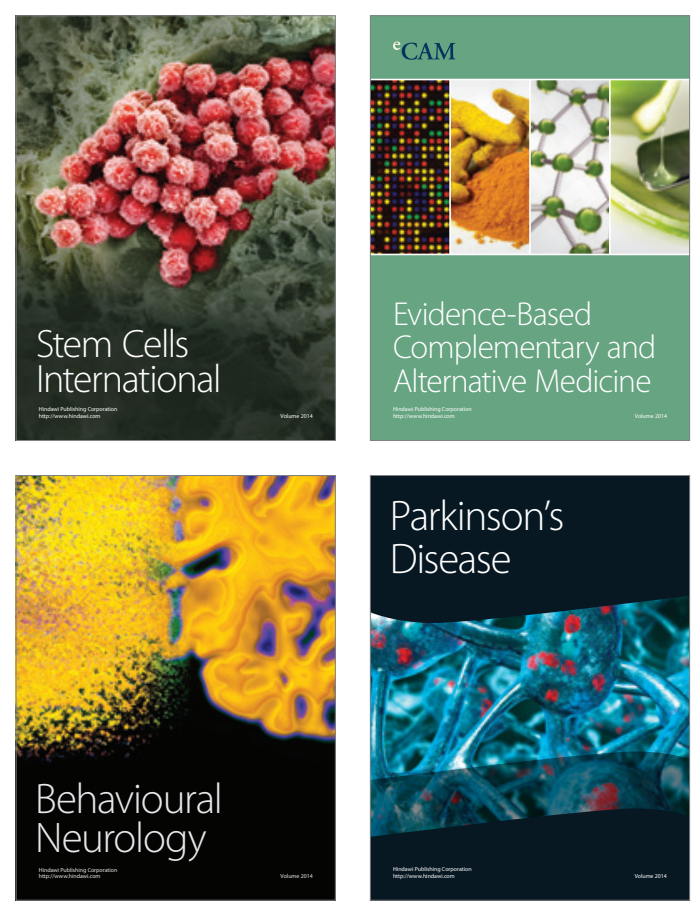

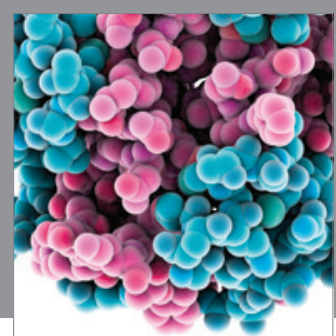

Journal of
Diabetes Research

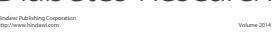

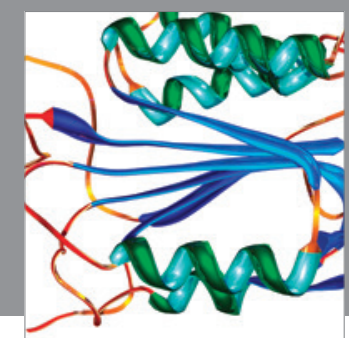

Disease Markers
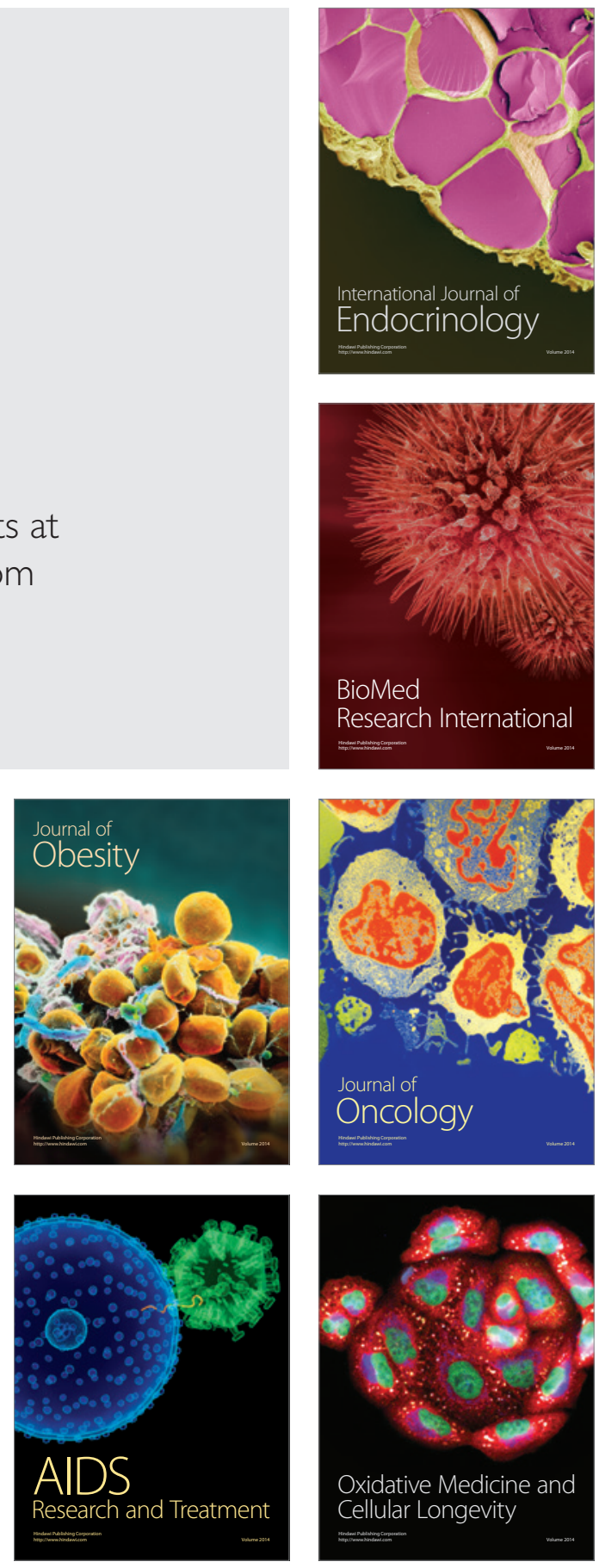